\title{
ON CERTAIN DOUBLY INFINITE SYSTEMS OF CURVES ON A SURFACE
}

\author{
BY C. H. ROWE
}

1. Introduction. A system of $\infty^{2}$ curves having been given on a surface, let us consider a variable curvilinear triangle bounded by three curves of the system. If we allow this triangle to shrink to a point $P$, the excess of the sum of its angles over $\pi$ (or, briefly, its excess) will tend to zero and will in general be an infinitesimal of the same order as the perimeter, the ratio of the excess to the perimeter tending to a limit which depends not only on the position of the point $P$ but also on the directions of the three curves through $P$ with which the sides tend to coincide. For special systems, however, it may happen that the excess is an infinitesimal of the same order as the area, as in the case where the system consists of the geodesics of the surface; or it may happen that the limiting value of the ratio of the excess to the perimeter depends only on the position of the point $P$. We are thus led to ask whether limiting properties of this kind are characteristic of any interesting systems of $\infty^{2}$ curves on a surface; and we shall consider from this point of view velocity systems, systems of hypergeodesics, and the systems that have been studied by Ogura under the name of $V$-systems.

2. Notation. We shall use orthogonal curvilinear coordinates on the surface, the element of length being given by $d s^{2}=E d u^{2}$ $+G d v^{2}$. A rotation in the tangent plane will be called direct if it agrees in sense with the rotation through a right angle from the positive direction of the $u$-curve ( $v=$ constant) to the positive direction of the $v$-curve. When a positive sense has been defined along a curve on the surface, the direction that is obtained by giving a direct rotation of one right angle to the positive tangent will be called the positive normal to the curve in the tangent plane.

We shall denote by $\theta$ the inclination (measured in the direct sense) of the positive direction of the curve to the positive direction of the $u$-curve; and we shall denote by $\rho$ the radius 
of geodesic curvature of the curve with the usual convention of sign, so that $\rho$ is positive if the center of geodesic curvature lies on the positive half of the normal in the tangent plane. The sign of $\rho$ thus depends on our choice of the positive direction along the curve.

For a triangle bounded by three curves on the surface we shall denote the lengths of the sides by $a_{1}, a_{2}, a_{3}$, the perimeter $a_{1}+a_{2}+a_{3}$ by $p$, and the excess by $e$.

3. Condition that $e / p$ should Tend to Zero. From the well known formula of Bonnet

$$
\int d \theta-\int \frac{d s}{\rho}=\iint K d S
$$

in which $K$ is the total curvature, we easily derive the expression

$$
e=\int \frac{d s}{\rho}+\iint K d S
$$

for the excess of a triangle bounded by three curves on the surface.* The curvilinear integral is taken around the boundary of the triangle in the direct sense, and this is the sense that we are to regard as the positive sense along the sides for the purpose of determining the value of the inclination $\theta$ and the sign of $\rho$.

We shall consider a triangle traced on the surface, and we shall allow it to shrink to a point $P$, so that the curves that form its sides tend to coincide with three curves through $P$ having distinct tangents there. The directions that we agreed to regard as positive along the sides of the triangle determine in the limit certain directions along the three curves through $P$, and, regarding these directions as positive, we shall denote by $\theta_{1}, \theta_{2}, \theta_{3}$ and by $\rho_{1}, \rho_{2}, \rho_{3}$ the values of $\theta$ and of $\rho$ for these curves at $P$.

If we assume that the curves that form the sides tend in a sufficiently regular manner to their limiting positions, we may write the approximate formula

$$
e=\sum \frac{a_{r}}{\rho_{r}}+o(p)
$$

\footnotetext{
* Darboux, Leçons sur la Thêorie Générale des Surfaces, vol. 3, p. 127.
} 
where the summation extends over the values $1,2,3$ of $r$, and where, in the usual notation, $o(p)$ denotes an error whose ratio to the perimeter tends to zero.

We shall now assume that the ratio of the excess to the perimeter tends to zero, so that

$$
\sum \frac{a_{r}}{\rho_{r}}=o(p) .
$$

If we combine this with the formulas

$$
\sum a_{r} \cos \theta_{r}=o(p), \quad \sum a_{r} \sin \theta_{r}=o(p),
$$

which we. obtain by projecting the sides of the triangle on the tangents to the coordinate curves, we see that, whatever the numbers $\lambda$ and $\mu$ are, we have

$$
\sum a_{r}\left(\frac{1}{\rho_{r}}-\mu \cos \theta_{r}+\lambda \sin \theta_{r}\right)=o(p) .
$$

If we choose $\lambda$ and $\mu$ so that two of the coefficients of $a_{1}, a_{2}, a_{3}$ in this sum are zero, the third must be zero also, because $a_{1}, a_{2}, a_{3}$ are infinitesimals of the same order as $p$. It follows that there exist numbers $\lambda$ and $\mu$ such that the three equations

$$
\frac{1}{\rho_{r}}=\mu \cos \theta_{r}-\lambda \sin \theta_{r} \quad(r=1,2,3)
$$

are true simultaneously.

This shows that the centers of geodesic curvature of the three curves through $P$ are collinear; for if we introduce rectangular cartesian coordinates in the tangent plane, taking the positive tangents to the $u$-curve and the $v$-curve as $x$-axis and $y$-axis respectively, the coordinates of these points are $-\rho_{r} \sin \theta_{r}$, $\rho_{r} \cos \theta_{r}$, and the equations (3) show that these points lie on the straight line whose equation is $\lambda x+\mu y=1$.

The proof of the converse of this result presents no difficulty, and we may thus state the following theorem:

When a triangle traced on a surface shrinks to a point, a necessary and sufficient condition that the ratio of the excess to the perimeter should tend to zero is that the three curves with which the sides tend to coincide should have their centers of geodesic curvature at the point collinear. 
We may notice a corollary which follows at once from this. If three curves through a point have their centers of geodesic curvature collinear, they retain this property when the surface is transformed conformally.

4. Velocity Systems. We shall now consider triangles bounded by curves belonging to a given system of $\infty^{2}$ curves on the surface, and we shall suppose that this system is determined by an equation of the form $1 / \rho=f(\theta, u, v)$. It follows easily from (2) that, when the triangle shrinks to a point, $e / p$ tends in general to a limit which depends on the position of this point and on the limiting directions of the sides of the triangle. We shall first ask what the nature of the system of curves must be in order that this limit should always be zero.

It will be clear at once from the theorem of the preceding paragraph that a necessary and sufficient condition for this is that the curves of the system that pass through any point should have their centers of geodesic curvature at the point collinear, or, in other words, that the equation that determines the system should be of the form

$$
\frac{1}{\rho}=\mu(u, v) \cos \theta-\lambda(u, v) \sin \theta .
$$

This condition means that the system of curves is a velocity system,* and we therefore have the following characteristic property of velocity systems:

$A$ system of $\infty^{2}$ curves on a surface has the property that the excess of any infinitesimal triangle bounded by curves of the system is an infinitesimal of higher order than the perimeter if, and only if, the system is a velocity system.

It will be seen that this result leads immediately to the theorem that in a conformal transformation of the surface a velocity system is transformed into a velocity system. $\dagger$

If in (1) we substitute the value of $\rho$ given by (4) and write

$$
\cos \theta d s=E^{1 / 2} d u, \sin \theta d s=G^{1 / 2} d v,
$$

we obtain by means of Green's theorem the formula

* E. Kasner, Natural families of trajectories: conservative fields of force, Transactions of this Society, vol. 10 (1909), pp. 201-219.

$\dagger$ See Kasner, loc. cit., p. 126. 


$$
e=\iint\left[K-\frac{1}{H} \frac{\partial}{\partial u}\left(\frac{\lambda H}{E^{1 / 2}}\right)-\frac{1}{H} \frac{\partial}{\partial v}\left(\frac{\mu H}{G^{1 / 2}}\right)\right] d S
$$

where

$$
H^{2}=E G
$$

We may therefore complete our result by adding that, for an infinitesimal triangle formed with curves of a velocity system, the ratio of the excess to the area tends to a limit which depends only on the position of the point to which the triangle ultimately reduces.

This limit is the integrand in the surface integral (5), and in order to interpret this expression we shall define a vector $\mathbf{F}$ in the tangent plane at each point $P$ of the surface such that its projections on the positive tangents to the $u$-curve and the $v$-curve at $P$ are $\lambda$ and $\mu$ respectively. If we denote the surface divergence of this vector by $\operatorname{div} F$, it will be seen that we may write

$$
\lim \frac{e}{A}=K-\operatorname{div} \mathbf{F},
$$

where $A$ is the area of the small triangle.

The vector $F$ has the property that the geodesic curvature of any curve of the system at $P$ is equal to the projection of $\mathbf{F}$ on the positive normal to the curve in the tangent plane.

If we recall the dynamical interpretation of a velocity system we may define the vector $F$ in another way. The velocity curves on a surface relative to a given field of force and to a given speed $v_{0}$ have the property that at each of its points a velocity curve osculates a trajectory of a particle which moves on the surface under the action of the force and has at the point a velocity of magnitude $v_{0}$. It will be seen that the vector $F$ associated with the velocity system is identical, except for a constant scalar factor, with the component of the force in the tangent plane.

We may notice that the condition that a velocity system should be an isogonal system is that $e$ should vanish for every triangle, and therefore that the vector $F$ should satisfy the equation $\operatorname{div} \mathbf{F}=K{ }^{*}$ We may also remark that the velocity systems for which the vector satisfies the equation $\operatorname{div} \mathbf{F}=0$

\footnotetext{
* See Kasner, loc. cit., p. 217.
} 
are the systems that have been mentioned by Darboux $\uparrow$ as sharing with the geodesics of the surface the property expressed in the theorem of Gauss on the excess of a geodesic triangle.

5. Hypergeodesics. If we are given a system of $\infty^{2}$ curves which is not a velocity system, it will in general be possible to ensure that the excess of an infinitesimal triangle bounded by curves of the system is negligible compared with the perimeter by imposing a suitable restriction on the limiting directions of the sides. We may thus ask whether we can characterize any known systems of curves by the particular form that this restriction takes. We shall show that for the systems that have been called hypergeodesics by Wilczynski* this restriction takes a specially simple characteristic form.

A system of hypergeodesics consists of the curves that satisfy the differential equation obtained by equating $d^{2} v / d u^{2}$ to a polynomial of the third degree in $d v / d u$ in which the coefficients are functions of $u$ and $v$; and it is easily seen that this is equivalent to saying that the equation

$$
\frac{1}{\rho}=f(\theta, u, v)
$$

that determines the system is of the form

$$
\frac{1}{\rho}=a \cos ^{3} \theta+b \sin ^{3} \theta+c \cos \theta+d \sin \theta,
$$

where $a, b, c, d$ are functions of $u$ and $v$.

In order that the relation $e=o(p)$ should be satisfied by a triangle which is about to shrink to a point $P$, the centers of geodesic curvature at $P$ of the curves of the system to which the sides tend must be collinear, and, with the notation of $\$ 3$, the condition for this is

$$
\frac{1}{\rho_{1}} \sin \left(\theta_{2}-\theta_{3}\right)+\frac{1}{\rho_{2}} \sin \left(\theta_{3}-\theta_{1}\right)+\frac{1}{\rho_{3}} \sin \left(\theta_{1}-\theta_{2}\right)=0 .
$$

If we replace the geodesic curvatures in this equation by their

$\dagger$ Darboux, Sur une série de lignes analogues aux lignes géodésiques, Annales de l'École Normale, (1), vol. 7 (1780), p 176.

* E. J. Wilczynski, Some generalizations of geodesics, Transactions of this Society, vol. 23 (1922), pp. 223-239. 
values as given by (6), we find after some simple reductions that our condition is equivalent to

$$
\tan \left(\theta_{1}+\theta_{2}+\theta_{3}\right)=\frac{a}{b}
$$

provided that the three curves through $P$ are distinct.

If we write

$$
\bar{\theta}=\frac{1}{3}\left(\theta_{1}+\theta_{2}+\theta_{3}\right),
$$

we may refer to the direction corresponding to the angle $\bar{\theta}$ as the mean direction of the sides of the infinitesimal triangle, and we notice that this direction depends only on the directions of the sides of the triangle and not on the direction of the $u$-curve from which the angles $\theta_{r}$ are measured. We may now say that, for a system of hypergeodesics, the condition that we have to impose on an infinitesimal triangle takes the form of a restriction placed on the mean direction of the sides; for the equation (7) requires that this direction should coincide with one of the six directions corresponding to the angles $\theta$ for which $\tan 3 \theta=a / b$.

We shall next show that this special form of the condition is characteristic of systems of hypergeodesics. Consider a system of curves determined by an equation $1 / \rho=f(\theta, u, v)$, which we may write $1 / \rho=f(\theta)$ without risk of confusion, and we shall show that, if the equation

$$
\theta_{1}+\theta_{2}+\theta_{3}=3 k \text { or } \quad \bar{\theta}=k
$$

where $k$ is a given function of $u$ and $v$, implies $e=o(p)$ or $f\left(\theta_{1}\right) \sin \left(\theta_{2}-\theta_{3}\right)+f\left(\theta_{2}\right) \sin \left(\theta_{3}-\theta_{1}\right)+f\left(\theta_{3}\right) \sin \left(\theta_{1}-\theta_{2}\right)=0$, then $f(\theta)$ has the form appropriate to a system of hypergeodesics.

We may assume without loss of generality that $k$ is zero, because we can clearly arrange that this is so by a suitable choice of our coordinate system. The assumption that we are making about the function $f(\theta)$ is now equivalent to saying that it is a solution of the functional equation

(8) $f(\theta+\phi) \sin (\theta-\phi)+f(-\theta) \sin (\theta+2 \phi)-f(-\phi) \sin (2 \theta+\phi)=0$.

It will be clear that, if $f(\theta)$ satisfies this equation, the function $g(\theta)$ defined by

$$
g(\theta)=f(\theta)-\left(b \sin ^{3} \theta+c \cos \theta+d \sin \theta\right)
$$


does so also, $b, c, d$ being any functions of $u$ and $v$. We shall determine $b, c, d$ so that $g(\theta)$ vanishes for the three values $0, \alpha, \beta$ of $\theta$, where $\alpha$ and $\beta$ are two arbitrarily chosen numbers whose ratio is irrational.

If we write $\phi=-\theta$ in (8), we see that any solution of this functional equation that vanishes for $\theta=0$ is an odd function. The function $g(\theta)$ is therefore an odd function, and the equation that it satisfies can consequently be written

$$
g(\theta+\phi) \sin (\theta-\phi)=g(\theta) \sin (\theta+2 \phi)-g(\phi) \sin (2 \theta+\phi) .
$$

Using this equation repeatedly, we see that the vanishing of $g(\alpha)$ and $g(\beta)$ implies that of $g(m \alpha+n \beta)$, where $m$ and $n$ are any two integers positive or negative. Since we can choose the integers $m$ and $n$ so that $m \alpha+n \beta$ is as close as we please to any given number, the function $g(\theta)$ must vanish identically, if we assume that it is continuous. The function $f(\theta)$ is therefore of the form (6) with $a=0$, and the system of curves is accordingly a system of hypergeodesics. We may therefore state the following theorem:

When a system of $\infty^{2}$ curves on a surface is a system of hypergeodesics, but in no other case, we can ensure that the excess of an infinitesimal triangle bounded by curves of the system is negligible compared with the perimeter by imposing a restriction on the mean direction of the sides.

6. V-systems. We shall examine lastly the conditions under which infinitesimal triangles bounded by curves of a given system on a surface have the property that the ratio of the excess to the perimeter depends only on the position of the point to which the triangle ultimately reduces.

With the notation that we have used before, the condition that, as a triangle shrinks to a point, $e / p$ should tend to a given limit $\eta$ is that the inequality

$$
\sum a_{r}\left(\frac{1}{\rho_{r}}-\eta\right)=o(p)
$$

should be satisfied. Arguing as we did in $\S 3$, we see that this implies the existence of two numbers $\lambda$ and $\mu$ such that the three equations

$$
\frac{1}{\rho_{r}}-\eta=\mu \cos \theta_{r}-\lambda \sin \theta_{r}, \quad(r=1,2,3),
$$


are true together. In order, therefore, that triangles formed from a given system of curves should have the limiting property in question, a relation of the type

$$
\frac{1}{\rho}=\eta(u, v)+\mu(u, v) \cos \theta-\lambda(u, v) \sin \theta
$$

must hold along the sides of every triangle.

The equation (9) shows that the system of curves must be such that the centers of geodesic curvature at any point $P$ of the curves that pass through $P$ lie on a conic one of whose foci is at $P$. Systems of this kind have been studied by Ogura* and have been named by him $V$-systems.

Moreover, the angle $\theta$ in the equation (9) corresponds to the direction in which the curve is described when a point moves in the direct sense around a triangle of which this curve forms a side, and, according to this equation, two distinct curves through a point correspond in general to the angles $\theta$ and $\theta+\pi$. This means that the equation indicates a certain sense along each curve and requires that, when the curve is used in forming a triangle, this sense should agree with the direct sense of description of the boundary of the triangle. We must consider only triangles for which this restriction is observed if the relation $e=p \eta(u, v)+o(p)$ is to be satisfied as the triangle shrinks to the point $(u, v)$.

The restriction that we are imposing on the triangles may be expressed otherwise by saying that the equation (9) decides for us which half of the normal to the curve in the tangent plane is to point inwards when the curve forms part of the boundary of a triangle.

Conversely, if we are given a $V$-system, we can choose for each curve of the system the direction along the normal in the tangent plane that will have to point inwards when the curve is used in forming a triangle, and we can make this choice (at least for a limited portion of the surface) in such a way that continuity is observed as we move along a curve or pass from curve to curve. If this is done, it will be seen without difficulty that triangles formed with curves of the system have the limiting property under discussion.

* K. Ogura, On certain systems of doubly infinite curves on a surface, Tôhoku Mathematical Journal, vol. 8 (1915), pp. 213-217. 
We may state our results in the following theorem:

$V$-systems, and no other systems, have the property that, for an infinitesimal triangle bounded by curves of the system, the ratio of the excess to the perimeter is a function of position, provided that we choose for each curve a direction along the normal in the tangent plane, having regard to continuity, and consider only triangles for which this direction points inwards.

We obtain as an immediate corollary to this a theorem given by Ogura which states that, when a surface is transformed conformally, a $V$-system is transformed into a $V$-system.

A simple illustration of our result is given by the $V$-system in the plane that consists of circles of constant radius $a$. The choice that we have to make amounts in this case to deciding whether the sides of a triangle shall have their convexities always turned outwards or always turned inwards, and, according as we adopt the first or the second of these alternatives, the excess of a triangle which conforms to our restriction is equal to $p / a$ or to $-p / a$.

This example suggests the problem of finding the most general system of curves on a surface such that the excess of any triangle bounded by curves of the system is equal to a constant multiple of the perimeter, provided only that a certain restriction as to sense is observed. It will be seen that the solution is given by the $V$-system determined by an equation of the form (9) where $\eta(u, v)$ is a constant and where the associated velocity system defined by the equation

$$
\frac{1}{\rho}=\mu(u, v) \cos \theta-\lambda(\quad v) \sin
$$

is an isogonal system.

Trinity College, Dublin 